University of Nebraska - Lincoln

DigitalCommons@University of Nebraska - Lincoln

Papers in Ornithology

Papers in the Biological Sciences

1961

\title{
Wintering Distribution Changes in Mallards and Black Ducks
}

Paul A. Johnsgard

University of Nebraska-Lincoln, pajohnsgard@gmail.com

Follow this and additional works at: https://digitalcommons.unl.edu/biosciornithology

Part of the Ornithology Commons

Johnsgard, Paul A., "Wintering Distribution Changes in Mallards and Black Ducks" (1961). Papers in Ornithology. 72.

https://digitalcommons.unl.edu/biosciornithology/72

This Article is brought to you for free and open access by the Papers in the Biological Sciences at DigitalCommons@University of Nebraska - Lincoln. It has been accepted for inclusion in Papers in Ornithology by an authorized administrator of DigitalCommons@University of Nebraska - Lincoln. 


\title{
Wintering Distribution Changes in Mallards and Black Ducks
}

\author{
PAUL A. JOHNSGARD \\ Department of Zoology, University of Nebraska, Lincoln
}

\begin{abstract}
Through the use of data compiled by the Audubon Society's annual Christmas Counts, an attempt has been made to trace the distributional changes of wintering Mallards and Black Ducks in the eastern states from 1900 to the present. During this period the Black Duck has increased relative to the Mallard in few if any states (the Great Lakes region), whereas the Mallard has increased nearly everywhere else (except in the extreme northeast), particularly in the southern coastal states. Deforestation and changes in land use are suggested as probable reasons for this trend, which is not likely to be reversed.
\end{abstract}

Although several rather spectacular shifts in the distributions of various American species of birds have become evident in recent years, the "colonization" of the eastern states by the Mallard (Anas platyrhynchos) has not been so generally recognized. The magnitude of this range extension has been great nevertheless, and the probable reasons for it have been discussed elsewhere (Johnsgard, 1959, 1961). Concomitant with the increase of the Mallard in the eastern states, the native Black Duck (Anas rubripes) has suffered a decline in numbers, and apparently for some of the same reasons as the Mallard has increased. Since this trend is not likely to be reversed in the future, it will be of interest to follow it carefully and thus possibly to predict the fate of the Black Duck.

Wing (1943) became interested in the ratios of Mallards to Black Ducks throughout the eastern states before the range shift was at all apparent, and calculated state ratios for the two forms on the basis of the data provided by the annual Audubon Society Christmas counts for the forty years 1900 to 1939 . He found that the average ratios for that period (which, because of the continuously increasing number of counts, is probably typical of the situation somewhat after the midpoint of 1920) indicated that the zone of equal ratios fell in a northsouth line between Michigan and western Florida. East of this line the Black Duck was markedly dominant over the Mallard and west of it the Mallard was equally dominant. Wing's data are presented in Table I, but they are converted from simple Mallard: Black Duck ratios to the alternative method of calculating the relative percentages of each form in the combined sample. This latter means of presenting ratio data has certain statistical advantages (Hickey, 1957). Arguments supporting the use of these data as unbiased estimates of wintering Mallard and Black Duck populations have been presented elsewhere (Johnsgard, 1959), and so will not be repeated here.

To test the possibility that these counts might indicate the degree to which the Mallard has moved eastward in recent years, it was de- 
cided to bring these calculations up to date, by collating the Christmas Count records for the twenty years 1940 to 1959 . Since the numbers of counts and counters has increased enormously since 1940, the data were divided into earlier (1940-1949) and later (1950-1959) periods. Again, although the averages for these total periods are presented (Table I), they probably reflect the existing conditions somewhat after the mid-points of the periods. It must be remembered too that changes in the ratios can be attributed both to Mallard increases in the East and to Black Duck decreases, rather than only to Mallard increases alone.

The data for the years 1940 to 1949 show some interesting differences from Wing's data for the preceding forty years. Of the states east of the Michigan to western Florida zone, one state (Florida) exhibits a majority of Mallards in the wintering population. Fourteen states exhibit what are probably significant reductions in the percentages of Black Ducks, with the most marked changes occurring in Florida, North Carolina, and Michigan. Only four states (Wisconsin, Kentucky, West Virginia and Virginia) exhibit noticeable increases in Black Duck ratios; of these West Virginia's data are probably not based on a sufficient sample. Since Wing's (1943) data did not include information on sample size it is impossible to evaluate these differences statistically.

The data for the years 1950 to 1959 bring to light some even more striking changes. Five states (Florida, Georgia, South Carolina, North Carolina and Ohio) east of the Michigan-western Florida zone exhibit majorities of Mallards in the samples. Eighteen states exhibit marked declines in Black Duck ratios from those presented by Wing. Only two states (Minnesota and Wisconsin) exhibit significant increases in Black Duck ratios over those of preceding years.

To provide a relatively up-to-date picture of the situation, data for the years 1958 to 1960 are also presented in Table I. Although in some cases the sample sizes are too small to be useful, in most a still greater decrease in Black Duck ratios is evident. It is astonishing to contemplate that in twenty years Delaware, for example, has undergone a shift in ratios from an almost pure Black Duck population to one in which Mallards are more abundant than Black Ducks. Other only slightly less remarkable examples are provided by the coastal states from North Carolina to Florida. On the other hand, the northern interior states have undergone less pronounced decreases, such as, for example, Michigan, Illinois, Indiana and Ohio. If a line delineating the zone of equality between Mallard and Black ratios were to be drawn today, it would still have to pass through Michigan at the north, but be deflected eastward to the south so as to pass through Virginia and North Carolina. Only in Maine and the Canadian maritime provinces may the wintering population still be considered "pure" Black Duck. It appears, therefore, that the Mallard has been invading the East by a "flanking" movement along the Gulf Coast states. It is hard to determine how rapidly the Mallard has colonized 
TABLE I.-Relative numbers of wintering Mallards and Black Ducks, based on Audubon Christmas Counts from 1900-1960

\begin{tabular}{|c|c|c|c|}
\hline State or Province & $\begin{array}{c}\text { Total } \\
\text { Black Ducks }\end{array}$ & $\begin{array}{c}\text { Total } \\
\text { Mallards }\end{array}$ & $\begin{array}{c}\text { Per cent } \\
\text { Black Duck }\end{array}$ \\
\hline $\begin{array}{r}\text { Nova Scotia } \\
1950-1959 \\
1958-1960\end{array}$ & $\begin{array}{l}5,962 \\
1,865\end{array}$ & $\begin{array}{l}2 \\
1\end{array}$ & $\begin{array}{l}99.97 \\
99.95\end{array}$ \\
\hline $\begin{array}{l}\text { Quebec } \\
1940-1949 \\
1950-1959 \\
1958-1960\end{array}$ & $\begin{array}{l}2,092 \\
6,314 \\
1,821\end{array}$ & $\begin{array}{r}81 \\
155 \\
50\end{array}$ & $\begin{array}{l}96.27 \\
97.60 \\
97.33\end{array}$ \\
\hline $\begin{array}{l}\text { Ontario } \\
1900-1939 \\
1940-1949 \\
1950-1959 \\
1958-1960\end{array}$ & $\begin{array}{r}9,370 \\
15,163 \\
7,261\end{array}$ & $\begin{array}{l}\ldots \ldots . \\
1,450 \\
7,936 \\
3,793\end{array}$ & $\begin{array}{l}97.51 \\
86.59 \\
65.36 \\
65.69\end{array}$ \\
\hline $\begin{array}{l}\text { Maine } \\
1940-1949 \\
1950-1959 \\
1958-1960\end{array}$ & $\begin{array}{l}1,773 \\
6,543 \\
5,102\end{array}$ & $\begin{array}{r}2 \\
43 \\
29\end{array}$ & $\begin{array}{l}99.89 \\
99.35 \\
99.43\end{array}$ \\
\hline $\begin{array}{c}\text { New Hampshir } \\
1950-1959 \\
1958-1960\end{array}$ & $\begin{array}{l}5,791 \\
1,736\end{array}$ & $\begin{array}{l}171 \\
234\end{array}$ & $\begin{array}{l}97.13 \\
88.12\end{array}$ \\
\hline $\begin{array}{l}\text { Vermont } \\
1940-1949 \\
1950-1959 \\
1958-1960\end{array}$ & $\begin{array}{l}272 \\
538 \\
170\end{array}$ & $\begin{array}{r}11 \\
12 \\
5\end{array}$ & $\begin{array}{l}96.11 \\
97.82 \\
97.14\end{array}$ \\
\hline $\begin{array}{c}\text { Massachusetts } \\
1900-1939 \\
1940-1949 \\
1950-1959 \\
1958-1960\end{array}$ & $\begin{array}{r}78,570 \\
117,499 \\
53,679\end{array}$ & $\begin{array}{r}472 \\
7,002 \\
4,606\end{array}$ & $\begin{array}{l}99.28 \\
98.52 \\
94.37 \\
90.38\end{array}$ \\
\hline $\begin{array}{c}\text { Rhode Island } \\
1900-1939 \\
1940-1949 \\
1950-1959 \\
1958-1960\end{array}$ & $\begin{array}{r}18,468 \\
18,759 \\
6,800\end{array}$ & $\begin{array}{l}101 \\
410 \\
221\end{array}$ & $\begin{array}{l}99.35 \\
99.45 \\
97.86 \\
96.85\end{array}$ \\
\hline $\begin{array}{r}\text { Connecticut } \\
1900-1939 \\
1940-1949 \\
1950-1959 \\
1958-1960\end{array}$ & $\begin{array}{l}\ldots \ldots . \\
19,398 \\
39,802 \\
15,269\end{array}$ & $\begin{array}{r}\ldots \ldots . \\
577 \\
13,646 \\
8,838\end{array}$ & $\begin{array}{l}94.31 \\
97.11 \\
74.47 \\
63.34\end{array}$ \\
\hline $\begin{array}{l}\text { New York } \\
\quad \text { (entire state } \\
1900-1939 \\
1940-1949 \\
1950-1959 \\
1958-1960\end{array}$ & $\begin{array}{r}135,376 \\
202,713 \\
65,891\end{array}$ & $\begin{array}{r}\ldots \ldots . \\
13,426 \\
30,563 \\
14,417\end{array}$ & $\begin{array}{l}96.12 \\
90.97 \\
86.90 \\
82.05\end{array}$ \\
\hline
\end{tabular}


TABle I.-(continued)

\begin{tabular}{|c|c|c|c|}
\hline State or Province & $\begin{array}{c}\text { Total } \\
\text { Black Ducks }\end{array}$ & $\begin{array}{c}\text { Total } \\
\text { Mallards }\end{array}$ & $\begin{array}{c}\text { Per cent } \\
\text { Black Duck }\end{array}$ \\
\hline $\begin{array}{l}\text { New York } \\
\text { (Long Islan } \\
1940-1949 \\
1950-1959 \\
1958-1960\end{array}$ & $\begin{array}{r}78,172 \\
125,489 \\
43,530\end{array}$ & $\begin{array}{r}4,990 \\
13,711 \\
7,276\end{array}$ & $\begin{array}{l}94.00 \\
90.15 \\
85.68\end{array}$ \\
\hline $\begin{array}{l}\text { New York } \\
\quad \text { (rest of stat } \\
1940-1949 \\
1950-1959 \\
1958-1960\end{array}$ & $\begin{array}{l}57,204 \\
77,224 \\
22,361\end{array}$ & $\begin{array}{r}8,436 \\
16,852 \\
7,141\end{array}$ & $\begin{array}{l}87.15 \\
82.09 \\
75.79\end{array}$ \\
\hline $\begin{array}{r}\text { New Jersey } \\
1900-1939 \\
1940-1949 \\
1950-1959 \\
1958-1960\end{array}$ & $\begin{array}{r}50,305 \\
226,744 \\
104,640\end{array}$ & $\begin{array}{r}3,922 \\
28,631 \\
10,542\end{array}$ & $\begin{array}{l}99.17 \\
92.76 \\
88.79 \\
90.85\end{array}$ \\
\hline $\begin{array}{r}\text { Pennsylvania } \\
1900-1939 \\
1940-1949 \\
1950-1959 \\
1958-1960\end{array}$ & $\begin{array}{l}63,933 \\
90,307 \\
20,456\end{array}$ & $\begin{array}{r}9 . \ldots \\
95,953 \\
21,043\end{array}$ & $\begin{array}{l}95.39 \\
86.52 \\
61.83 \\
49.29\end{array}$ \\
\hline $\begin{array}{l}\text { Delaware } \\
1900-1939 \\
1940-1949 \\
1950-1959 \\
1958-1960\end{array}$ & $\begin{array}{r}21,777 \\
118,489 \\
31,288\end{array}$ & $\begin{array}{r}634 \\
85,175 \\
42,432\end{array}$ & $\begin{array}{l}99.08 \\
97.17 \\
58.18 \\
42.44\end{array}$ \\
\hline $\begin{array}{l}\text { Maryland } \\
1900-1939 \\
1940-1949 \\
1950-1959 \\
1958-1960\end{array}$ & $\begin{array}{r}23,309 \\
102,184 \\
43,482\end{array}$ & $\begin{array}{r}3,108 \\
60,343 \\
30,719\end{array}$ & $\begin{array}{l}94.08 \\
88.23 \\
62.87 \\
58.60\end{array}$ \\
\hline $\begin{array}{c}\text { Washington, I } \\
1940-1949 \\
1950-1959 \\
1958-1960\end{array}$ & $\begin{array}{l}1,854 \\
7,619 \\
1,717\end{array}$ & $\begin{array}{r}376 \\
2,170 \\
449\end{array}$ & $\begin{array}{l}83.14 \\
77.83 \\
79.27\end{array}$ \\
\hline $\begin{array}{l}\text { Virginia } \\
1900-1939 \\
1940-1949 \\
1950-1959 \\
1958-1960\end{array}$ & $\begin{array}{r}15,557 \\
68,736 \\
26,531\end{array}$ & $\begin{array}{r}2,599 \\
31,259 \\
13,706\end{array}$ & $\begin{array}{l}67.53 \\
85.67 \\
68.74 \\
65.94\end{array}$ \\
\hline $\begin{array}{c}\text { North Carolin } \\
1900-1939 \\
1940-1949 \\
1950-1959 \\
1958-1960\end{array}$ & $\begin{array}{r}3,133 \\
28,830 \\
16,849\end{array}$ & $\begin{array}{r}1,831 \\
29,522 \\
16,593\end{array}$ & $\begin{array}{l}90.13 \\
63.10 \\
49.41 \\
50.38\end{array}$ \\
\hline
\end{tabular}


TABLE I.-(continued)

\begin{tabular}{|c|c|c|c|}
\hline State or Province & $\begin{array}{c}\text { Total } \\
\text { Black Ducks }\end{array}$ & $\begin{array}{c}\text { Total } \\
\text { Mallards }\end{array}$ & $\begin{array}{c}\text { Per cent } \\
\text { Black Duck }\end{array}$ \\
\hline $\begin{array}{c}\text { South Garolina } \\
1900-1939 \\
1940-1949 \\
1950-1959 \\
1958-1960\end{array}$ & $\begin{array}{l}3,232 \\
3,169 \\
1,483\end{array}$ & $\begin{array}{r}\ldots \ldots \\
1,534 \\
49,281 \\
32,341\end{array}$ & $\begin{array}{r}71.35 \\
67.84 \\
6.04 \\
4.59\end{array}$ \\
\hline $\begin{array}{l}\text { Georgia } \\
1900-1939 \\
1940-1949 \\
1950-1959 \\
1958-1960\end{array}$ & $\begin{array}{r}21,266 \\
169 \\
210\end{array}$ & $\begin{array}{r}\ldots \ldots . \\
10,882 \\
2,258 \\
984\end{array}$ & $\begin{array}{r}70.58 \\
66.10 \\
6.96 \\
17.59\end{array}$ \\
\hline $\begin{array}{l}\text { Florida } \\
1900-1939 \\
1940-1949 \\
1950-1959 \\
1958-1960\end{array}$ & $\begin{array}{c}\ldots \ldots \\
1,188 \\
1,142 \\
304\end{array}$ & $\begin{array}{l}1,854 \\
4,164 \\
1,296\end{array}$ & $\begin{array}{l}65.98 \\
29.09 \\
21.52 \\
19.00\end{array}$ \\
\hline $\begin{array}{l}\text { Ohio } \\
1900-1939 \\
1940-1949 \\
1950-1959 \\
1958-1960\end{array}$ & $\begin{array}{r}33,983 \\
105,670 \\
65,153\end{array}$ & $\begin{array}{r}22,266 \\
150,582 \\
78,541\end{array}$ & $\begin{array}{l}66.89 \\
60.32 \\
41.23 \\
45.34\end{array}$ \\
\hline $\begin{array}{c}\text { West Virginia } \\
1900-1939 \\
1940-1949 \\
1950-1959 \\
1958-1960\end{array}$ & $\begin{array}{r}\ldots 22 \\
1,321 \\
642\end{array}$ & $\begin{array}{r}35 \\
674 \\
427\end{array}$ & $\begin{array}{l}88.01 \\
94.67 \\
66.21 \\
60.06\end{array}$ \\
\hline $\begin{array}{l}\text { Kentucky } \\
1900-1939 \\
1940-1949 \\
1950-1959 \\
1958-1960\end{array}$ & $\begin{array}{r}7,216 \\
39,902 \\
6,912\end{array}$ & $\begin{array}{r}11,083 \\
375,891 \\
23,875\end{array}$ & $\begin{array}{r}15.31 \\
42.74 \\
9.60 \\
22.45\end{array}$ \\
\hline $\begin{array}{l}\text { Tennessee } \\
1900-1939 \\
1940-1949 \\
1950-1959 \\
1958-1960\end{array}$ & $\begin{array}{l}4,477 \\
6,044 \\
8,288\end{array}$ & $\begin{array}{r}33,844 \\
354,969 \\
176,772\end{array}$ & $\begin{array}{r}19.54 \\
11.68 \\
1.67 \\
4.48\end{array}$ \\
\hline $\begin{array}{l}\text { Alabama } \\
1940-1949 \\
1950-1959 \\
1958-1960\end{array}$ & $\begin{array}{r}4,257 \\
5,024 \\
89\end{array}$ & $\begin{array}{r}19,380 \\
59,720 \\
380\end{array}$ & $\begin{array}{r}18.02 \\
7.70 \\
18.98\end{array}$ \\
\hline $\begin{array}{l}\text { Mississippi } \\
1900-1939 \\
1940-1949 \\
1950-1959 \\
1958-1960 \\
\end{array}$ & $\begin{array}{r}96 \\
2 \\
0\end{array}$ & $\begin{array}{r}1,119 \\
11,621 \\
178\end{array}$ & $\begin{array}{r}14.56 \\
7.90 \\
0.02 \\
0.00\end{array}$ \\
\hline
\end{tabular}


TABLE I.-(continued)

\begin{tabular}{|c|c|c|c|}
\hline State or Province & $\begin{array}{c}\text { Total } \\
\text { Black Ducks }\end{array}$ & $\begin{array}{c}\text { Total } \\
\text { Mallards }\end{array}$ & $\begin{array}{c}\text { Per cent } \\
\text { Black Duck }\end{array}$ \\
\hline $\begin{array}{l}\text { Michigan } \\
1900-1939 \\
1940-1949 \\
1950-1959 \\
1958-1960\end{array}$ & $\begin{array}{r}7,800 \\
15,275 \\
2,146\end{array}$ & $\begin{array}{r}6,656 \\
11,990 \\
1,868\end{array}$ & $\begin{array}{l}71.75 \\
53.91 \\
56.02 \\
53.46\end{array}$ \\
\hline $\begin{array}{l}\text { Indiana } \\
1900-1939 \\
1940-1949 \\
1950-1959 \\
1958-1960\end{array}$ & $\begin{array}{r}26,425 \\
68,372 \\
4,935\end{array}$ & $\begin{array}{r}93,660 \\
1,442,230 \\
69,187\end{array}$ & $\begin{array}{r}21.37 \\
22.03 \\
4.53 \\
6.65\end{array}$ \\
\hline $\begin{array}{l}\text { Illinois } \\
1900-1939 \\
1940-1949 \\
1950-1959 \\
1958-1960\end{array}$ & $\begin{array}{r}51,625 \\
74,486 \\
11,112\end{array}$ & $\begin{array}{r}2,311,226 \\
1,410,753 \\
290,805\end{array}$ & $\begin{array}{l}8.52 \\
2.19 \\
5.01 \\
3.68\end{array}$ \\
\hline $\begin{array}{l}\text { Wisconsin } \\
1900-1939 \\
1940-1949 \\
1950-1959 \\
1958-1960\end{array}$ & $\begin{array}{r}7,175 \\
12,543 \\
3,452\end{array}$ & $\begin{array}{l}14,794 \\
41,853 \\
22,118\end{array}$ & $\begin{array}{l}10.10 \\
32.38 \\
23.06 \\
13.50\end{array}$ \\
\hline $\begin{array}{l}\text { Minnesota } \\
1900-1939 \\
1940-1949 \\
1950-1959 \\
1958-1960\end{array}$ & $\begin{array}{r}36 \\
682 \\
31\end{array}$ & $\begin{array}{l}3,337 \\
8,705 \\
4,463\end{array}$ & $\begin{array}{l}0.07 \\
1.07 \\
7.26 \\
0.69\end{array}$ \\
\hline $\begin{array}{l}\text { Iowa } \\
1900-1939 \\
1940-1949 \\
1950-1959 \\
1958-1960\end{array}$ & $\begin{array}{r}6 . . \\
210 \\
207\end{array}$ & $\begin{array}{l}29,745 \\
45,724 \\
13,949\end{array}$ & $\begin{array}{l}0.22 \\
0.22 \\
0.46 \\
1.46\end{array}$ \\
\hline $\begin{array}{l}\text { Missouri } \\
1900-1939 \\
1940-1949 \\
1950-1959 \\
1958-1960\end{array}$ & $\begin{array}{r}\ldots \ldots . \\
460 \\
163 \\
93\end{array}$ & $\begin{array}{r}94,516 \\
275,485 \\
168,045\end{array}$ & $\begin{array}{l}0.70 \\
0.48 \\
0.06 \\
0.06\end{array}$ \\
\hline $\begin{array}{l}\text { Arkansas } \\
1900-1939 \\
1940-1949 \\
1950-1959 \\
1958-1960\end{array}$ & $\begin{array}{r}1 \\
136 \\
32\end{array}$ & $\begin{array}{r}871 \\
479,094 \\
156,625\end{array}$ & $\begin{array}{l}0.33 \\
0.12 \\
0.03 \\
0.02\end{array}$ \\
\hline $\begin{array}{l}\text { Louisiana } \\
1900-1939 \\
1940-1949 \\
1950-1959 \\
1958-1960\end{array}$ & $\begin{array}{r}\ldots \ldots \\
490 \\
194 \\
54\end{array}$ & $\begin{array}{r}20,626 \\
28,228 \\
4,581\end{array}$ & $\begin{array}{l}7.57 \\
2.33 \\
0.68 \\
1.16\end{array}$ \\
\hline
\end{tabular}


TABLE I.-(continued)

\begin{tabular}{cccc}
\hline State or Province & $\begin{array}{c}\text { Total } \\
\text { Black Ducks }\end{array}$ & $\begin{array}{c}\text { Total } \\
\text { Mallards }\end{array}$ & $\begin{array}{c}\text { Per cent } \\
\text { Black Duck }\end{array}$ \\
\hline North Dakota & 1 & 1,977 & 0.05 \\
$1940-1949$ & 0 & 1,661 & 0.00 \\
$1950-1959$ & 0 & 99 & 0.00 \\
$1958-1960$ & & & \\
South Dakota & $\ldots \ldots$. & 0.21 \\
$1900-1939$ & 10 & 340,265 & 0.003 \\
$1940-1949$ & 35 & 484,132 & 0.01 \\
$1950-1959$ & 10 & 300,664 & 0.003 \\
$1958-1960$ & & & \\
Nebraska & 0 & 475 & 0.00 \\
$1940-1949$ & 0 & 119,635 & 0.00 \\
$1950-1959$ & 0 & 226,468 & 0.00 \\
$1958-1960$ & & & \\
Kansas & 0 & 18,457 & 0.07 \\
$1940-1949$ & 13 & 278,104 & 0.01 \\
$1950-1959$ & 23 & 58,712 & 0.007 \\
$1958-1960$ & 4 & & \\
Oklahoma & & & 0.02 \\
$1940-1949$ & 65 & 271,791 & 0.02 \\
$1950-1959$ & 42 & 219,765 & 0.01 \\
$1958-1960$ & 5 & 34,058 & \\
Texas & & & 0.01 \\
$1900-1939$ & $\ldots \ldots$ & 371,396 & 0.01 \\
$1940-1949$ & 29 & 134,715 & \\
$1950-1959$ & 17 & & \\
$1958-1960$ & & & \\
\hline \hline
\end{tabular}

the eastern states as a breeding bird, but counts made by federal and state biologists during the breeding season indicate that Mallard-Black Duck ratios in the northern states and provinces during the period 1948-1956 closely parallel the wintering ratios of those areas for the same period (Johnsgard, 1959).

It would appear that the Mallard has in the past few decades made enormous inroads into what formerly was predominantly Black Duck territory, whereas the reverse is true only to a very limited extent in the Great Lakes region. Probably this can be attributed to the great changes in land use, and the deforestation, which have occurred in the East during the present century, and which have had the effect of decreasing Black Duck breeding habitat while at the same time increasing Mallard habitat. Whether sufficient preservation and regeneration of the northeastern forests will occur to permit the Black Duck to retain a stronghold is impossible to predict, but it appears likely that the Black Duck, through hybridization with the much larger 
Mallard gene pool and through the constant reduction of its breeding habitat, may eventually disappear as a distinct entity from our fauna.

Acknowledgment.-This study was done at Cornell University under the support of fellowships from the Cornell University Graduate School and the National Science Foundation. I would like to express my appreciation to these agencies as well as to Dr. C. G. Sibley, my graduate committee chairman.

\section{REFERENGES}

Hrakey, J. J. 1957. Editorial note. J. Wildl. Mgmt., 21:347.

JoHNSGARD, P. A. 1959. Evolutionary relationships among the North American mallards. Ph.D. Thesis, Cornell University. 153 p.

1961. Evolutionary relationships among the North American mallards. Auk, 78:3-43.

WrNG, L. 1943. Relative distribution of Mallard and Black Duck in winter. Ibid., 60 :438-439. 